\title{
A Comparison of Two Video laryngoscopes: Truview and Kingvision in Routine Airway Management
}

Namazi IJ ${ }^{1 *}$, Sahni $\mathrm{S}^{2}$

${ }^{1}$ MD Anesthesia, Professor and Head of Department of Anesthesia, Dr. DY Patil Medical College and Hospital, Kolhapur, Maharashtra, India.

${ }^{2}$ MD Anesthesia, Dr. DY Patil Medical College and Hospital, Kolhapur, Maharashtra, India.

Abstract
Background: With advances in technology, intubation using video laryngoscopy has been gaining popularity, particularly
in patients with difficult airways or as rescue devices in failed intubation attempts. Their routine use is, however, an un-
common occurrence. This clinical trial was carried out to support their use in elective airway management.
Objective: To evaluate the effectiveness of kingvision video laryngoscope (KVL) in comparison with the Truview video
laryngoscope (TVL) in routine airway management.
Methods: We studied 60 subjects requiring orotracheal intubation during general anaesthesia for elective surgeries. They
were randomly assigned into two groups to undergo tracheal intubation using either a TVL (n=30) or KVL (n=30)
.Characteristics and consequences of airway management were evaluated. The primary outcomes were the percentage of
glottic opening (POGO) and ease of intubation and secondary outcomes were Cormack and Lehane (CL) grading, TTI
(time taken for intubation) and successful placement of endotracheal tube (ETT).
Results: Both the VLs were comparable with respect to primary and secondary outcomes except TTI which was more
with KVL.
Conclusion: We opine that both TVL and KVL provide a better laryngoscopic view in routine airway management, but
KVL does not have an extra benefit over TVL otherwise, further indicating the need for more experience with the use
of a KVL.
Keywords: Laryngoscopes; Airway Management; Intubation.

\section{*Corresponding Author:}

Namazi IJ,

MD Anesthesia, Professor and Head of Department of Anesthesia, Dr. DY Patil Medical College and Hospital, Kolhapur, Maharashtra, India. E-mail: drismailnamazi@gmail.com

Received: June 25, 2014

Accepted: July 22, 2014

Published: July 24, 2014

Citation: Namazi IJ, Sahni S (2014) A Comparison of Two Video laryngoscopes: Truview and Kingvision in Routine Airway Management. Int J Anesth Res. 2(5), 48-52. doi: http://dx.doi.org/10.19070/23322780-1400013

Copyright: Namazi IJ C 2014 This is an open-access article distributed under the terms of the Creative Commons Attribution License, which permits unrestricted use, distribution and reproduction in any medium, provided the original author and source are credited.

\section{Introduction}

Practitioners involved in airway management now have a plethora of tools often purported as rescue devices after failed direct laryngoscopy (DL). Video larygoscopes (VLs) are often useful as either a primary tool for intubation or as a rescue tool if immediately available during a difficult intubation.[1] However, prohibitively high costs and poor portability have prevented their being immediately available in many intubation situations and locations.

There is currently a paucity of clinical research comparing dif- ferent types of VLs, particularly when used in patients and not in manikins.

The TVL and KVL are two novice VLs that are portable as well as cost effective.

KVL is a new VL which provides indirect view of the glottis (figure 1). It consists of reusable anti-reflective display and a single use ergonomic blade/handle. Currently there are two available blade designs: disposable size 3 channeled blade and disposable size 3 standard blade. The distal lens has an anti-fog coating.[2] There is limited data available about its clinical efficacy.

The TVL (Truphatek Israel) is a Macintosh-type blade with an optical lens attached (figure 2) which provides a better view of the glottis. [3]

The present study evaluates and compares the efficacy of these two VLs for tracheal intubation based on the primary outcomes POGO and ease of intubation and secondary outcomes CL grading, TTIand successful placement of ETT.

\section{Methods}

After obtaining an approval from the Ethics Committee and written, informed consent from all the 60 patients of either sex, aged between 18 and 65 years, belonging to ASA physical status class I or II, undergoing elective surgery and requiring oral intubation under general anaesthesia were included. Patients having anticipated difficult airway Mallampati Class III or IV ,thyromental 
Figure 1. KVL

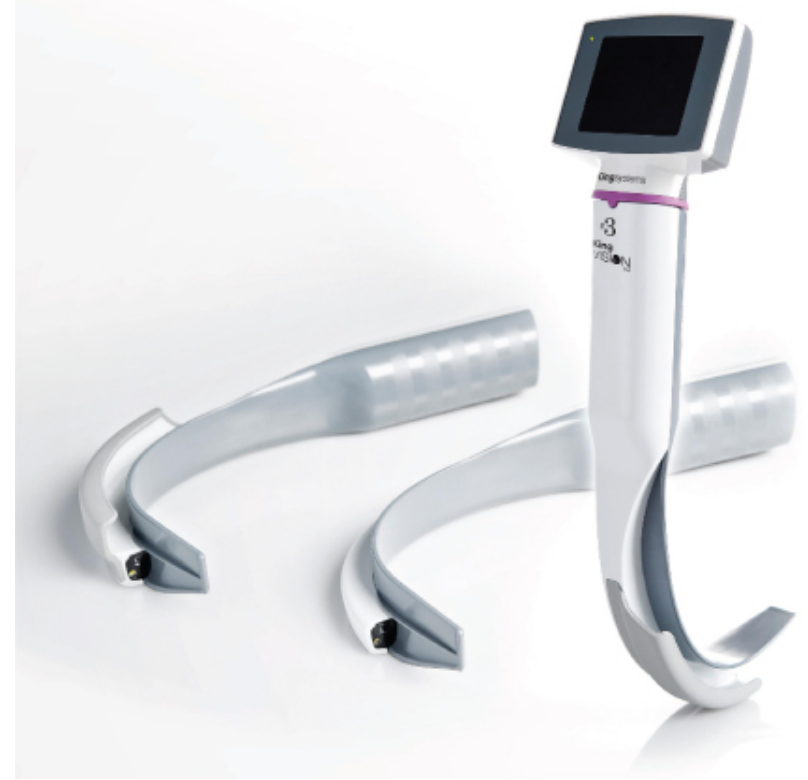

Figure 2. TVL

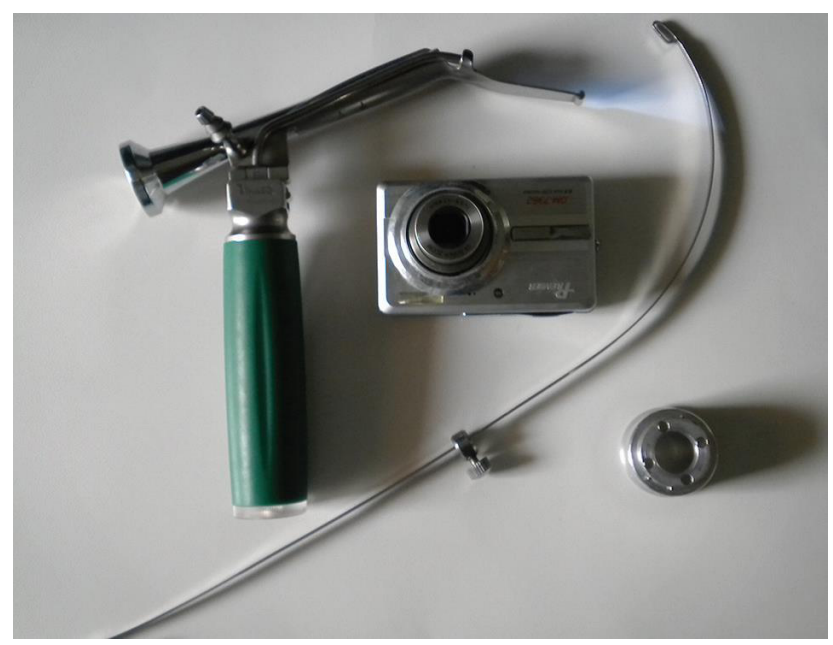

distance less than $6 \mathrm{~cm}$, inter-incisor distance less than $3 \mathrm{~cm}$, history of difficult airway, cervical spine injury, risk of regurgitation, emergency surgery and body mass index $(\mathrm{BMI})>30 \mathrm{Kg} \mathrm{m}^{-2}$ were excluded from the study.

All patients underwent a preanaesthetic evaluation and were randomized into two groups with 30 patients each; group $\mathrm{T}$-using TVL \& group K-using KVL by computer generated random number table and sealed envelope method.

All patients were premedicated with oral diazepam $10 \mathrm{mg}$ and ranitidine $150 \mathrm{mg}, 2$ hours prior to surgery. In the operating room standard monitors were attached and intravenous (IV) access obtained and an IV infusion was started. The anaesthesia work station was checked and prepared for intubation. A single use Portex cuffed ETT7 for females and 8 for males was used. A hockey shaped stylet was used with Truview and channeled number three blade KVL with the ETT loaded on it was kept ready. Patients were pre oxygenated with $100 \% \mathrm{FiO}_{2}$ with face mask. Anaesthesia was induced with glycopyrrolate $4 \mathrm{ug} \mathrm{kg}{ }^{-1}$, fentanyl $2 \mathrm{ug} \mathrm{kg}^{-1}$ and propofol $2 \mathrm{mg} \mathrm{kg}{ }^{-1} \mathrm{IV}$. Succinylcholine $1.5 \mathrm{mg} \mathrm{kg}^{-1}$ facilitated orotracheal intubation performed in neutral position with either laryngoscope by a laryngoscopist who had performed 50 successful intubations with both laryngoscopes in a manikin. After successful intubation and confirmation of ETT placement by capnograph, anaesthesia was maintained with oxygen, nitrous oxide, isoflourane and injection vecuronium $0.1 \mathrm{mg} \mathrm{kg}^{-1} \mathrm{IV}$.

With both the laryngoscopes, the view of the glottis at laryngoscopy was scored according to the CL grading and POGO score ( $0 \%$ when glottis is not seen and $100 \%$ when entire glottis is seen). [4] No laryngeal manipulation or external pressure was given to improve the laryngoscopic view or improve the score.

The TTI was defined as the time taken from insertion of laryngoscope blade into the mouth until evidence obtained of the presence of $\mathrm{CO}_{2}$ in the exhaled breath after intubation. If necessary, the anesthetist assistant was allowed to apply external pressure or to do laryngeal manipulation to assist intubation.Number of attempts was noted. Patients were ventilated with 100\% oxygen between attempts at laryngoscopy and intubation so that no patient was allowed to desaturate below 95\%. After three attempts at intubation or intubation requiring more than 120 seconds with assigned blade, patients were intubated using Macintosh blade and was considered as failed intubation.

The ease of intubation as observed by anaesthesiologist was graded as given in appendix 1. [5]

\section{Statistical analysis}

Based on a previous report by Lange et al. [6], and assuming a likely difference in mean time for intubation of $10 \mathrm{~s}$ with a SD of $12 \mathrm{~s}$ between groups, as a prior ipower analysis revealed that a group size of 24 was needed to detect a difference with a power of 0.8 , at ana-level of 0.05 . To minimise the impact of data loss, we planned to enrol 30 patients to the study. Mean and standard deviation were used to describe parametric data and patient numbers and percentages to describe categorical data. Statistical significance of parametric data was determined by the Student $t$ test and categorical data by the Chi-square test $\mathrm{P}<0.05$ was considered statistically significant.

\section{Results}

A total of 60 patients were enrolled in the study. There were no exclusions after recruitment. Demographic data included variables of gender, age and weight which were comparable between the two groups (Table 1). Preanaesthetic evaluation and airway assessment were also comparable (Table 2).

Intubation parameters were assessed and compared. CL grading of $\mathrm{I}$ was noted in $80 \%$ of patients in T group and $66.66 \%$ in $\mathrm{K}$ group.CL grading of II was noted in $20 \%$ patients in T group and $33.33 \%$ patients in $\mathrm{K}$ group.

The POGO score was comparable in both the groups, Difference 
CONSORT 2010 Flow Diagram

\section{Enrollment}
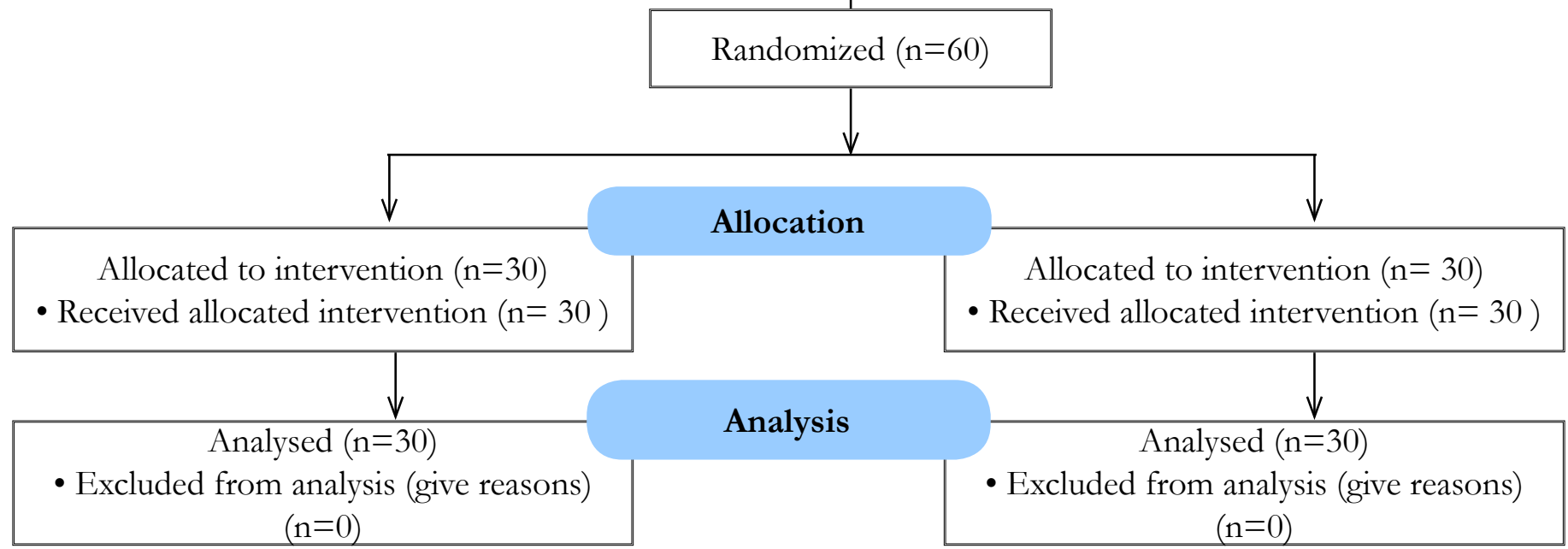

in TTI was extremely statistically significant which was less than 0.0001 .

$100 \%$ successful intubation rate was noted in both the groups. Patients intubated in first attempt in group T were 93.3\% and 86.6 $\%$ in group $\mathrm{K}$ whereas intubation in second attempt was $6.6 \%$ for group $\mathrm{T}$ and $13.3 \%$ for group $\mathrm{K}$.

The ease of intubation was grade I (easy) in $73.3 \%$ for group T and $66.6 \%$ for group K, grade 2 in $20 \%$ of patients for both the groups, grade 3 in $6.6 \%$ for group $\mathrm{T}$ and $13.3 \%$ for group $\mathrm{K}$ and no group had grade 4 level of intubation.

\section{Discussion}

Difficulty in placing an endotracheal tube is an important cause of morbidity and mortality in the operating room, emergency department, intensive care setting and in out-of -hospital resuscitation. [7]

Table 1. Demographic data of patients in the study

\begin{tabular}{|l|l|l|l|}
\hline Variable & Truview & Kingvision & $\boldsymbol{P}_{\text {value }}$ \\
\hline Age ( mean years) $\mathbf{\text { SD }}$ & $55.35 \pm 5.26$ & $54.05 \pm 5.41$ & $0.34[\mathrm{NS}]^{*}$ \\
\hline Weight (mean kilogram) $\mathbf{E S D}$ & $57.37 \pm 7.42$ & $59.05 \pm 5.12$ & $0.29[\mathrm{NS}]^{*}$ \\
\hline Gender (\%) & & & \\
\hline Female & $8(26.6)$ & $10(33.33)$ & \\
\hline Male & $22(73.33)$ & $20(66.66)$ & \\
\hline ASA Grade (\%) & & & \\
\hline I & $8(26.6)$ & $12(40)$ & \\
\hline II & $22(73.33)$ & $18(60)$ & \\
\hline
\end{tabular}

NS* -non significant ASA - American Society of Anaesthesiologists; SD - Standard deviation

Table 2. Preoperative airway assessment parameters of the patients in the study

\begin{tabular}{|l|l|l|l|}
\hline Variable & Truview & Kingvision & $\boldsymbol{P}_{\text {value }}$ \\
\hline Mallampati Class (\%) & & & \\
\hline $\mathbf{1}$ & $7(23.33)$ & $6(20)$ & \\
\hline $\mathbf{2}$ & $23(76.66)$ & $24(80)$ & \\
\hline Thyromental Distance (mean mm) $\mathbf{\pm S D}$ & $62.95 \pm 3.09$ & $63.525 \pm 2.96$ & $0.39[\mathrm{NS}]^{*}$ \\
\hline Interincisor Distance (mean mm) $\mathbf{\mathbf { S D }}$ & $34.3 \pm 2.28$ & $34.5 \pm 1.39$ & $0.68[\mathrm{NS}]^{*}$ \\
\hline
\end{tabular}

NS *-non significant 
Table 3. Study data (primary and secondary outcome measures) of the patients in the study

\begin{tabular}{|l|l|l|l|}
\hline Variable & Truview & Kingvision & $\boldsymbol{P}_{\text {value }}$ \\
\hline CL grade (\%) & & & \\
\hline I & $24(80)$ & $20(66.6)$ & \\
\hline II & $6(20)$ & $10(33.3)$ & \\
\hline TTI (mean seconds SD) & 32.6 & 40 & $<0.0001[\mathrm{~S}]^{\wedge}$ \\
\hline POGO (IQR) & $100(100-100)$ & $100(60-100)$ & {$[\mathrm{NS}]^{*}$} \\
\hline Ease of Intubation (I/II/III/IV) & $22 / 6 / 2 / 0$ & $20 / 6 / 4 / 0$ & \\
\hline Number of Attempts (1/2/3) & $28 / 2 / 0$ & $26 / 4 / 0$ & \\
\hline Failures & 0 & 0 & \\
\hline
\end{tabular}

CL-Cormack and Lehane grading; POGO-percentage of glottic opening; TTI-time taken for intubation IQR-interquartile range; $\mathrm{NS}{ }^{*}$-non significant; $\mathrm{S}^{\wedge}$-significant

Recently, the combination of the fiber optic bronchoscope and the laryngoscope led to the development of VLs, providing a video based view of the glottic opening, with or without additional guidance of the tube towards the tracheal opening. [8]

Video laryngoscopy is the major technological advancement that attempts to produce a view of the laryngeal inlet independent of the line of sight and improves success of tracheal intubation. As it obviates the need to align the oral, pharyngeal and tracheal axes, thereby obtaining a better laryngeal view and subsequent tracheal intubation easier to perform.[9] There have been limited studies comparing different types of VLs, majority of which are manikin studies. [10,11,12,13]

Moreover, many of these devices have received little if any independent evaluation. The need to determine the clinical utility of these devices, ideally before their widespread adoption into clinical practice, is clear if we are to avoid a situation where a poorly designed device contributes to the failure to perform tracheal intubation, leading to patient harm. [14]

This prospective randomized controlled trial was conducted to assess the utility of the KVL in routine adult airway management which is a relatively new device with very few studies in comparison to more well established TVL.

In our study, we have reported CL grading of I and II in both the groups.In addition to CL grading, the POGO score was also included as a measure of laryngeal view in our study. The POGO score is more sensitive than the CL grading and has been shown in previous studies to have good intra-and inter-observer reliability [15]. We reported a good POGO score in both the groups. POGO score obtained with VLs is higher than DLs. [16]

Difficulty in intubation despite good glottis visualization is a problem reported with most VLs. Most VLs can achieve a better view of the glottis and have a similar success rate.In our study, we found that visualization of the vocal cords was excellent, but the introduction of the tube was challenging in certain cases. Majority of cases in both the groups reported easy intubation. In $20 \%$ cases (grade 2) in each group we used manoeuvres like ELOM, slight withdrawal of VL blade, manipulation and redirection of ETT after rotation so that it enters the glottis, in cases where it was directed towards the pyriform fossa. These resulted in a successful intubation in the first attempt. We have also used the 'hockey-stick' J-curvature at the end of the tracheal tube for TVL as described by Sun and colleagues, [17]which helps to maneuver the ETT into the glottis. Those patients who required a second attempt in both the groups in spite of all the above manoeuvers (6.6\% in group $\mathrm{T}$ and $13.3 \%$ in group $\mathrm{K}$ ), we used optimization of blade position during re insertion in the oral cavity.

From the various studies done on VLs which shows that anaesthesiologists have reported difficulties in advancing the tube towards the glottis, we think that with sufficient experience in using these devices this problem can be easily overcome.

There are conflicting reports regarding TTI with VL. Some studies say that VLs are associated with a better laryngoscopic view but require a longer TTI. $[16,18]$ and some say that the time to tracheal intubation is not different between the VLs and DL for orotracheal intubation.[19]

Our mean TTI was 32.6 seconds for TVL and 40 seconds for KVL.

A study comparing Macintosh (MAC)and KVL showed overall median (range) intubation times (sec) were 16.9 (8.0-60.0) with the MAC and 20.5 (7.2-60.0) with the KV. Success rate with the MAC was $(91.4 \%)$ and KVL (86.6\%). Esophageal intubation with the MAC occurred in 18 of 186 attempts, whereas no incidents of esophageal intubation occurred with the KVL. The authors concluded that The KVL facilitated intubation by novice personnel without incidence of esophageal intubation. However, intubation times and success rates were similar to the values obtained with the MAC.[20]

Video laryngoscopy presents a new approach for the management of difficult and rescue laryngoscopy and has the additional potential to enhance the educationof novices. $[21,22,23]$ It has become a widely accepted method of learning the techniques of the airway management.[24]

An important potential advantage of the KVL is that it has a single-use disposable blade. This removes concerns regarding the potential for multi-use intubation devices to facilitate transmission of prions $[25,26]$ which are thought to be responsible for causing variant Creutzfeldt-Jakob disease. There are difficulties in ensuring that all proteinaceous material has been removed from reusable laryngoscope blades during cleaning and sterilization. $[27,28]$ In recognition of these concerns, the guidelines of the Association of Anaesthetists of Great Britain and Ireland state 
that 'single use intubation aids' should be used where possible.[29]

\section{Limitation}

This is a preliminary trial in a small patient population and in order to provide a more comprehensive evaluation of the King Vision the sample size should be increased.

We cannot comment on its use in anticipated difficult airway and further clinical studies are needed before establishing its use in difficult airway scenarios.

It is beyond the purview of the study to compare hemodynamic variables during intubation.

\section{Conclusion}

During this study, use of both the VLs resulted in a good glottic view with a good success rate of orotracheal intubation. In spite of a statistically significant difference in T'TI the KVL scored highly in overall performance even though the operators had no previous experience in use of this type of VL. The VLs are also good for training and help the anaesthetic assistant to apply glottis view optimizing manoeuvers if needed. Being portable and economical their use can be expanded to cover a wider variety of scenarios both in and out of hospital settings after further clinical trials.

\section{References}

[1] Aziz MF, Healy D, Kheterpal S, RongweiF,Dillman D,et al (2011) Routine clinical practice effectiveness of the Glidescope in difficult airway management: an analysis of 2004 Glidescope intubations, complications, and failures from two institutions. Anesthesiology; 114 (1):34-41

[2] King VISION Available from http://www.KingSystems.com | www. OwnTheAirway.com [Last accessed on 2014 march10].

[3] Matsumoto, Sanae ,Asai , Takashi, Shingu ,et al (2006) Truview Video Laryngoscope in Patients with Difficult Airways. Anesthesia\& Analgesia; $103: 492-3$

[4] Cook TM, Levitan RM, Ochrroch A, Hollander J (2000) POGO score Can J Anesth;47:477-8

[5] Arino JJ, Velasco JM, Gasco C, Lopez-Timoneda F (2003) Straight blades improve visualization of the larynx while curved blades increase ease of intubation: A comparison of the Macintosh, Miller, McCoy, Belscope and Lee-Fiberview blades. Can J Anaesth;50:501-6

[6] Lange M, Frommer M, Redel A (2009) Comparison of the Glidescope and Airtraq optical laryngoscopes in patients undergoing direct microlaryngoscopy. Anaesthesia; 64: 323-8

[7] Norris AM, Hardman JG, Asai T (2011) A firm foundation for progress in airway management. Br J Anaesth;106(5):613-6

[8] Lorenz Theiler, Kristina Hermann, Patrick Schoettker, Georges Savoldelli, Natalie Urwyler,et al (2013) Swiss video-intubation trial evaluating videolaryngoscopes in a simulated difficult airway scenario: study protocol for a multicentre prospective randomized controlled trial in Switzerland, 14:94
[9] Cooper RM, Pacey JA, Bishop MJ, McCluskey SA (2005) Early clinical experience with a new video laryngoscope (GlideScope) in 728 patients. Can J Anaesth;52:191-8.

[10] Maassen R, van Zundert A (2010) Comparison of the C-MAC video laryngoscope with the Macintosh, Glidescope and Airtraq laryngoscopes in easy and difficult laryngoscopy scenarios in manikins. Anaesthesia; 65: 955

[11] McElwain J, Malik MA, Harte BH, Flynn NM, Laffey JG (2010) Comparison of the C-MAC video laryngoscope with the Macintosh, Glidescope, and Airtraq laryngoscopes in easy and difficult laryngoscopy scenarios in manikins. Anaesthesia; 65: 483-9 14

[12] Sharma DJ, Weightman WM, Travis A (2010) Comparison of the Pentax Airway Scope and McGrath Video laryngoscope with the Macintosh laryngoscope in tracheal intubation by anaesthetists unfamiliar with video laryngoscopes: a manikin study. Anaesth Intensive Care; 38: 39-42

[13] Tan BH, Liu EHC, Lim RTC, Liow LMH, Goy RWL (2009) Ease of intubation with the GlideScope or Airway Scope by novice operators in simulated easy and difficult airways-a manikin study. Anaesthesia; 64: 187-90

[14] McElwain J, Malik MA, Harte BH,Flynn NM, Laffey JG (2010) Comparison of the C-MAC video laryngoscope with the Macintosh, Glidescope, and Airtraq laryngoscopes in easy and difficult laryngoscopy scenarios in manikins. Anaesthesia; 65: 483-9.

[15] Ochroch EA, Hollander JE, Kush S, Shofer FS, Levitan RM (1999) Assessment of laryngeal view: Percentage of glottic opening score vs Cormack and Lehane grading. Can J Anaesth;46:987-90

[16] Timanaykar RT, Anand LK, Palta S (2011) A randomized controlled study to evaluate and compare Truview blade with Macintosh blade for laryngoscopy and intubation under general anesthesia. J Anaesthesiol ClinPharmacol;27:199-204

[17] Sun DA, Warriner CB, Parsons DG, Klein R, Umedaly HS, et al (2005). The GlideScope video laryngoscope: randomized clinical trial in 200 patients. $\mathrm{Br}$ J Anaesth; 94: 381-4

[18] Barak M, Philipchuck P, Abecassis P, Katz Y (2007) A comparison of the Truview blade with the Macintosh blade in adult patients. Anaesthesia; 62: 827-31

[19] Li JB, Xiong YC, Wang XL (2007) An evaluation of the TruView EVO2 laryngoscope. Anaesthesia; 62: 940-3.

[20] Akihisa Y, Maruyama K, Koyama Y, Yamada R, Ogura A, et al (2014). Comparison of intubation performance between the King Vision and Macintosh laryngoscopes in novice personnel: a randomized, crossover manikin study. J Anesth;28(1):51-7.

[21] You JS, Park S, Chung SP, Park YS, Park JW (2009) The usefulness of the GlideScope video laryngoscope in the education of conventional tracheal intubation for the novice. Emerg Med J, 26:109-111

[22] Low D, Healy D, Rasburn N (2008) The use of the BERCI DCI Video Laryngoscopefor teaching novices direct laryngoscopy and tracheal intubation. Anaesthesia, 63:195-201.

[23] Kaplan MB, Ward DS, Berci G (2002) A new video laryngoscope-an aid tointubation and teaching. J Clin Anesth, 14:620-626

[24] Ray DC, Billington C, Kearns PK, Kirkbride R, Mackintosh K, et al (2009). A comparison of McGrath and Macintosh laryngoscopes in novice users: a manikin study. Anaesthesia;64:1207-10

[25] W Lowe PR, Engelhardt T (2001) Prion-related diseases and anaesthesia. Anaesthesia; 56: 48527

[26] Will RG, Ironside JW, Zeidler M (1996) A new variant of Creutzfeldt-Jakob disease in the UK. Lancet; 347: 921

[27] Miller DM, Youkhana I, Karunaratne WU, Pearce A (2001) Presence of protein deposits on 'cleaned' re-usable anaesthetic equipment. Anaesthesia;56: 1069-7229

[28] Hirsch N, Beckett A, Collinge J, Scaravilli F, Tabrizi S, et al (2005) Lymphocyte contamination of laryngoscope blades-a possible vector for transmission of variant Creutzfeldt-Jakob disease.Anaesthesia; 60: 664-7

[29] Infection Control in Anaesthesia. London: The Association of Anaesthetists of Great Britain and Ireland, 2002

\section{APPENDIX 1}

Grade 1: Intubation easy

Grade 2: Intubation requiring an increased anterior lifting force and assistance to pull the right corner of the mouth upwards to increase space

Grade 3: Intubation requiring multiple attempts and a curved stylet

Grade 4: Failure to intubate with the assigned Laryngoscope 